\title{
SOME GENERAL CLASSES OF COMATCHING GRAPHS
}

\author{
E.J. FARRELL and S.A. WAHID \\ Department of Mathematics \\ The University of the West Indies \\ St. Augustine, Trinidad \\ (Received May 6, 1985)
}

ABSTRACT. Some sufficient conditions are given for two graphs to have the same matching polynomial (comatching graphs). Several general classes of comatching graphs are given. Also, techniques are discussed for extending certain pairs of comatching graphs to larger pairs of comatching graphs.

KEY WORDS AND PHRASES. Matching polynomial, comatching graphs, weight, chains, cycles, attaching graphs to other graphs.

1980 AMS SUBJECT CLASSIFICATION CODE. 05A99, 05C99.

1. INTRODUCTION.

The graphs considered here will be finite and without loops or multiple edges. Let $\mathrm{G}$ be such a graph. We define a matching in $\mathrm{G}$ to be a spanning subgraph of $G$, whose components are nodes and edges only. Let us associate with each node in $G$, an indeterminate or weight $\mathrm{w}_{1}$, and with each edge, a weight $\mathrm{w}_{2} \cdot$ Also, let us associate with each matching in $G$ with $k$ edges, the weight $w_{1}^{p-2 k} w_{2}{ }^{k}$. Then, the matching polynomial of $\mathrm{G}$ is

$$
M(G ; \underline{w})=\sum_{k} a_{k} w_{1}^{p-2 k} w_{2}^{k},
$$

where $a_{k}$ is the number of matchings in $G$ with $k$ edges, $w$ is a weight vector $\left(w_{1}, w_{2}\right)$, and the summation is taken over all values of $k$. The basic results on matching polynomials are given in Farre11 [1].

We define two graphs to be comatching if and only if they have the same matching polynomial. In this paper, we investigate non-isomorphic comatching graphs. As far as we know, no investigation has been made into this interesting property of graphs. We will give some general results on comatching graphs. Then we will introduce various classes of comatching graphs. In many cases, we will give results concerning the construction of pairs of larger comatching graphs from certain "basic" pairs of comatching graphs (comatching pairs).

Let us denote the node set of $G$ by $V(G)$ and the edge set by $E(G)$. Let $A \subseteq V(G)$. Then $G-A$ will denote the graph obtained from $G$ by removing the nodes in $A$. When $A$ is a singleton $\{a\}$, we will write $G$ - a. Let $H$ be a subgraph of 
of $G$. Then $G-H$ will denote the graph obtained from $G$ by removing the nodes in H. For brevity, we will write $m(Q)$ for $M(G ; \underline{w})$.

2. SOME GENERAL RESULTS.

The following lemma is taken from [1]. It is called the fundamental theorem for matching polynomials.

LEMMA 1. Let $G$ be a graph and $x y$ an edge in $G$, where $x, y \in V(G)$. Let $G^{\prime}$ be the graph obtained from $G$ by deleting $x y$ and $G^{\prime \prime}$ the graph $G-\{x, y\}$. Then $M(G ; \underline{w})=M\left(G^{\prime} ; \underline{w}\right)+w_{2} M\left(G^{\prime \prime} ; \underline{w}\right)$.

We will refer to the algorithm implied by this lemma, as the reduction process. $G^{\prime}$ will be called the reduced graph and G", the incorporated graph. The following is called the Component Theorem. It was also proved in [1].

LEIMA 2. Let $G$ be a graph consisting of $r$ components $G_{1}, G_{2}, \ldots, G_{r}$ Then

$$
\mathrm{m}(\mathrm{G})=\underset{i=1}{\mathrm{r}} \mathrm{m}\left(G_{i}\right) \text {. }
$$

An immediate consequence of the definitions given in Section 1 is the following theorem.

THEOREM 1. Two graphs are comatching if and only if they have the same numbers of the same kinds of matchings.

Trivially, we have the following corollary in which $\bar{G}$ represents the complement of $G$.

COROLLARY 1.1. (G, G) is a comatching pair, for all graphs G. Also, if $G$ is selfcomplementary, $(G, \bar{G})$ is a comatching pair.

The following are the smallest non-trivial comatching pair $\left(\mathrm{H}_{1}, \mathrm{H}_{2}\right)$ and the smallest connected comatching pair $\left(\mathrm{H}_{3}, \mathrm{H}_{4}\right)$.
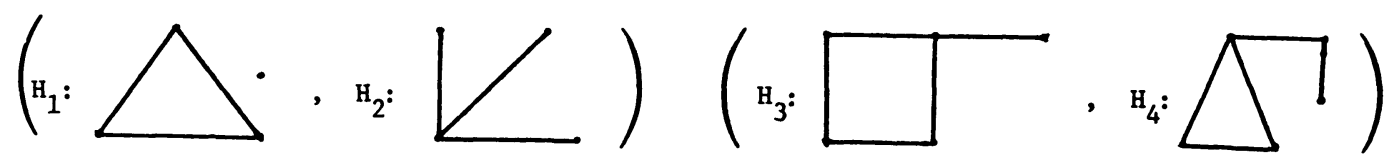

Figure 1

Notice that $\overline{\mathrm{H}}_{3}=\mathrm{H}_{4}$ and therefore $\mathrm{H}_{3}$ is not self-complementary. $\mathrm{H}_{3}$ is the smallest (connected) non-selfcomplementary graph with the property that $\left(\mathrm{H}_{3}, \overline{\mathrm{H}}_{3}\right)$ is a comatching pair. It can be easily confirmed that

$$
m\left(H_{1}\right)=m\left(H_{2}\right)=w_{1}^{4}+3 w_{1}^{2} w_{2}
$$

and that

$$
m\left(H_{3}\right)=m\left(H_{4}\right)=w_{1}^{5}+5 w_{1}^{3} w_{2}+4 w_{1} w_{2}^{2} \text {. }
$$

Let $G$ be a graph. Two nodes $u$ and $v$ in $G$ are called pseudosimilar if $G-u$ and $G-v$ are isomorphic, but no automorphism of $G$ maps $u$ onto $v$. We will denote by $G_{a}+H_{b}$ the graph formed from two connected graphs $G$ and $H$ 
by identifying node $a$ of $G$ with node $b$ of $H$.

THEOREM 2. Let $G$ be a graph with pseudosimilar nodes $u$ and $v$. Let $H$ be a graph containing a node $x$. Then

$$
m\left(G_{u}+H_{x}\right)=m\left(G_{v}+H_{x}\right) \text {. }
$$

PROOF. We will prove the result by induction on the valency $d(x)$ of node $x$. Suppose that $\mathrm{d}(\mathrm{x})=0$. Then $\mathrm{H}$ is a node and in this case we get

$$
\mathrm{G}_{\mathrm{u}}+\mathrm{H}_{\mathrm{x}}=\mathrm{G}_{\mathrm{v}}+\mathrm{H}_{\mathrm{x}}=\mathrm{G}
$$

and the result follows trivially. Let $d(x)=1$ and let $y$ be the node adjacent to node $x$. Apply the reduction process to $G_{u}+H_{x}$ using edge $x y$. Then $G^{\prime}$ (see Theorem 1) will consist of two components, $G$ and $H-x$. G" will consist of the components $\mathrm{G}-\mathrm{u}$ and $\mathrm{H}-\{\mathrm{x}, \mathrm{y}\}$. Hence by using the Component Theorem we get

$$
m\left(G_{u}+H_{x}\right)=m(G) m(H-x)+w_{2} m(G-u) m(H-\{x, y\}) .
$$

Apply the reduction process to $\mathrm{G}_{\mathrm{v}}+\mathrm{H}_{\mathrm{x}}$ by deleting edge $\mathrm{xy}$. Then $\mathrm{G}^{\prime}$ will consist of the components $G$ and $H-x$. G" will consist of the components $G-v$ and $\mathrm{H}-\{\mathrm{x}, \mathrm{y}\}$. Therefore we get

$$
m\left(G_{v}+H_{x}\right)=m(G) m(H-x)+w_{2} m(G-v) m(H-\{x, y\}) .
$$

But from the definition of pseudosimilar nodes,

$$
\begin{aligned}
\mathrm{G}-\mathrm{u} & \equiv \mathrm{G}-\mathrm{v} \\
\therefore \mathrm{m}(\mathrm{G}-\mathrm{u}) & =\mathrm{m}(\mathrm{G}-\mathrm{v}) .
\end{aligned}
$$

Hence from Equations (2.1) and (2.2), we get

$$
m\left(G_{u}+H_{x}\right)=m\left(G_{v}+H_{x}\right) \text {. }
$$

Let us assume that the result holds for all graphs $H$ with $d(x)<n$, and let $d(x)=n$ in $H$. Apply the reduction process to $G_{u}+H_{x}$ by deleting edge $x y$, where $y$ is one of the nodes in $H$ which is adjacent to node $x$. Then $G^{\prime}$ will be the graph $\mathrm{G}_{\mathrm{u}}+\mathrm{H}_{\mathrm{x}}^{\prime}$, where $\mathrm{H}^{\prime}$ is the graph obtained from $\mathrm{H}$ by deleting edge $\mathrm{xy}$. $\mathrm{G}^{\prime \prime}$ will consist of two components, $G-u$ and $H-\{x, y\}$. Hence

$$
m\left(G_{u}+H_{x}\right)=m\left(G_{u}+H_{x}^{\prime}\right)+w_{2} m(G-u) m(H-\{x, y\}) \text {. }
$$

Similarly, by applying the reduction process to $\mathrm{G}_{\mathrm{v}}+\mathrm{H}_{\mathrm{x}}$, we get

$$
m\left(G_{v}+H_{x}\right)=m\left(G_{v}=H_{x}^{\prime}\right)+w_{2} m(G-v) m(H-\{x, y\}) \text {. }
$$

But from the induction hypothesis,

$$
m\left(G_{u}+H_{x}^{\prime}\right)=m\left(G_{v}+H_{x}^{\prime}\right) \text {. }
$$

Therefore from Equations (2.3) and (2.4), we get

$$
m\left(G_{u}+H_{x}\right)=m\left(G_{v}+H_{x}\right) \text {. }
$$

Hence the result holds for $d(x)=n$. It follows by the Principle of Induction, that the result is true for all $n$.

Theorem 2 is a useful result for constructing comatching pairs, since the graphs $\mathrm{G}_{\mathrm{u}}+\mathrm{H}_{\mathrm{x}}$ and $\mathrm{G}_{\mathrm{v}}+\mathrm{H}_{\mathrm{x}}$ will generally be non-isomorphic.

3. A GENERAL TECHNIQUE FOR CONSTRUCTING COMATCHING GRAPHS.

Let $\mathrm{G}$ and $\mathrm{H}$ be connected graphs. We attach $\mathrm{H}$ to $\mathrm{G}$ by identifying a node $x$ of $H$ with a node $y$ of $G$. In the resulting graph, $G$ and $H$ will be subgraphs 
with exactly one node in common - the node $z$ formed in the identification process. $z$ will be called the node of attachment. Let us denote $G(H)$, the graph obtained from a given graph G, by attaching in the same manner, a copy of the graph $H$ to each of the nodes of $G$. It is clear that if $G$ contains $p_{1}$ nodes and $q_{1}$ edges, and $H$ contains $\mathrm{p}_{2}$ nodes and $\mathrm{q}_{2}$ edges, then $\mathrm{G}(\mathrm{H})$ will contain $\mathrm{p}_{1} \mathrm{p}_{2}$ nodes and $\left(\mathrm{q}_{1}+\right.$ $\mathrm{p}_{1} \mathrm{q}_{2}$ ) edges. If $\mathrm{H}$ is attached to only some of the nodes of $\mathrm{G}$, the resulting graph will be denoted by $G^{*}(H)$.

By a chain, we will mean a tree with nodes of valencies 1 and 2 only. The chain with $n$ nodes will be denoted by $P_{n}$. The followinglemma gives a simple technique for constructing a larger comatching pair, starting with a given comatching pair, which satisfies certain conditions.

LEMMA 3. Let $(A, B)$ be a comatching pair. Suppose that there exist nodes a and $b$ in $A$ and $B$ respectively, such that $(A-a, B-b)$ is also a comatching pair. Then $\left(\mathrm{P}_{2}(\mathrm{~A}), \mathrm{P}_{2}(\mathrm{~B})\right)$ is a comatching pair, when $a$ and $b$ are used in the attachment process.

PROOF. Apply the reduction process to $P_{2}(A)$ by deleting the edge belonging to $P_{2}$. The reduced graph will consist of two components, each being the graph A. The incorporated graph will consist of two copies of A - a. We therefore get

$$
m\left(P_{2}(A)\right)=m(A)^{2}+w_{2} m(A-a)^{2} .
$$

Similarly, we get

$$
m\left(P_{2}(B)\right)=m(B)^{2}+w_{2} m(B-b)^{2} .
$$

Since $(A, B)$ and $(A-a, B-b)$ are comatching pairs,

$$
m(A)=m(B) \text { and } m(A-a)=m(B-b) \text {. }
$$

Hence the result follows from Equations (3.1) amd (3.2).

The following theorem gives a generalization of the lemma. It can be easily proved by induction on the number of nodes in the chain.

THEOREM 3. Let $(A, B)$ be a comatching pair satisfying the conditions of Lemma 3. Then $\left(P_{n}(A), P_{n}(B)\right)$ is also a comatching pair, when the attachments are made using nodes $a$ and $b$.

It is unnecessary to attach $A$ and $B$ to every node of $P_{n}$ in order to obtain a comatching pair. This is implied by the following theorem, which is a further generalization of the lemma.

THEOREM 4. Let $(A, B)$ be a comatching pair satisfying the conditions of Lemma 3 . Then $\left(P_{n}^{*}(A), P_{n}^{*}(B)\right)$ is also a comatching pair, where the attachments are made to the same nodes of $P_{n}$ in forming the two graphs $P_{n}^{*}(A)$ and $P_{n}^{*}(B)$, and nodes $a$ and $b$ respectively are used in all the attachments. PROOF. We can apply the reduction process to $\mathrm{P}_{n}^{*}(A)$ by always deleting edges incident to nodes of attachment, until all the reduced and incorporated graphs are either graphs of the form $P_{r}(A)$ or $A-a$. The reduction process can then be applied to $P_{n}^{*}(B)$ in the Identical manner. The result will then follow from Lemma 1 , the Component Theorem 
and Theorem 3. $\square$

Theorem 4 can be generalized even further. Consider the graph $G^{*}(A)$. We can apply the reduction process to this graph until the reduced and incorporated graphs are of the form $\mathrm{P}_{\mathrm{r}}^{*}(\mathrm{~B})$ or $\mathrm{B}-\mathrm{b}$. Then, by using Lemma 3, the Component Theorem and Theorem 4, we obtain the following general result.

THEOREM 5. Let $(A, B)$ be a comatching pair such that $(A-a, B-b)$ is also a comatching pair, where $a$ and $b$ are nodes in $A$ and $B$ respectively. Then $\left(G^{*}(A)\right.$, $\left.G^{*}(B)\right)$ is also a comatching pair, when the attachments are made using nodes a and $b$ of $A$ and $B$ respectively.

Theorem 5 provides us with a general construction technique for large families of comatching graphs. The following is a comatching pair (A,B) satisfying the conditions of Lemma 3 .

A:

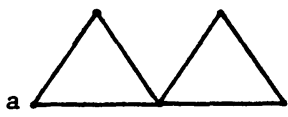

B : $\mathrm{b}$

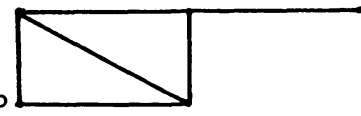

Figure 2

It can be eaily confirmed that

$$
m(A)=m(B)=w_{1}^{5}+6 w_{1}^{3} w_{2}+5 w_{1} w_{2}^{2}
$$

and that

$$
m(A-a)=m(B-b)=w_{1}^{4}+4 w_{1}^{2} w_{2}+w_{2}^{2} \text {. }
$$

The following theorem shows that instead of attaching the graphs $A$ and $B$ (of Lemma 3) to other graphs, we could instead attach other graphs to $A$ and $B$, in order to construct new comatching pairs.

THEOREM 6. Let $(A, B)$ be a comatching pair satisfying the conditions of Lemma 3 . Let $A^{*}(G)$ and $B^{*}(G)$ be the graphs obtained by attaching a graph $G$ to $A$ and $B$ respectively. Then $\left(A^{*}(G), B^{*}(G)\right)$ is a comatching pair.

PROOF. Apply the reduction process to $A^{*}(G)$ by deleting one of the edges of $G$ which is incident to the node of attachment - node $z$. Let $G_{1}^{\prime}$ be the reduced graph. The incorporated graph will consist of two components $G-y$ and $A-a$, where $y$ is the node used to attach $G$ to $A$. We can apply the reduction process to $G_{1}^{\prime}$ and to subsequent $G_{i}^{\prime}$ in the same manner until the reduced graph becomes disconnected with two components $G-y$ and $A$. Hence we will get

$$
m\left(A^{*}(G)\right)=m(G-y) m(A)+d_{y} m(G-y) m(A-a),
$$

where $d_{y}$ is the valency of node $y$ in $G$.

By applying the reduction process to $B^{*}(G)$, In an Identical manner, we get

$$
m\left(B^{*}(G)\right)=m(G-y) m(B)=d_{y} m(G-y) m(B-b) \text {. }
$$

Since $m(A)=m(B)$ and $m(A-a)=m(B-b)$, the result follows from Equations (3.3) and $(3.4)$.

4. COMATCHING COMPLEXES OF CHAINS AND CYCLES.

We will denote by $G_{r, s}$, the graph formed by attaching the chain $P_{r}$ (using an endnode) to the cycle $\mathrm{C}_{\mathrm{s}}$ with $\mathrm{s}$ nodes. 
THEOREM 7. ( $\left.G_{a, b}, G_{b-1, a+1}\right)$ is a comatching pair, for all positive integers a and b.

PROOF. Apply the reduction process to each graph in turn, by deleting an edge which is incident to the node of attachment. In both cases, the reduced graph will be $P_{a+b-1}$ and the incorporated graph will consist of two components $P_{a-1}$ and $P_{b-2}$. Hence the result follows from Lemma 1 .

Let $x$ and $y$ be the nodes of $G_{a, b}$ and $G_{b-1, a+1}$ respectively, which are adjacent to the node of attachment. Then it can be easily confirmed that $G_{a, b}-x$ will be $P_{a+b-2}$ and that $G_{b-1, a+1}-y$ will also be $P_{a+b-2}$. Hence these graphs satisfy the conditions of Lemma 3. Theorem 7 therefore gives us a general technique, not only for constructing comatching pairs, but for constructing graphs which satisfy Lemma 3 . Hence we can construct comatching complexes of chains and cycles, by using Theorem 5 .

The following theorem shows that the graph consisting of the components. ${ }_{r}$ and $\mathrm{C}_{\mathrm{r}+1}$ has the same matching polynomial as the chain $\mathrm{P}_{\mathrm{r}+1}$. It can be proved by appropriate applications of the reduction process.

THEOREM 8. For all, integers $\mathrm{r}$,

$$
m\left(P_{r}\right) m\left(C_{r+1}\right)=m\left(P_{2 r}+1\right)
$$

5. A GENERAL COMATCHING QUADRUPLE.

We will give a technique for constructing four non-isomorphic graphs with the same matching polynomial 1.e., a comatching quadruple. Let $(A, B)$ be a comatching pair, satisfying the conditions of Lemma 3 . Let $G$ be a graph containing two nodes $x$ and $y$. Construct four graphs as follows. $\mathrm{H}_{1}$ is the graph obtained by attaching a graph A (using node a) to $G$, at the nodes $x$ and $y$. Similarly $H_{2}, H_{3}$ and $H_{4}$ are the graphs formed by attaching to $G$, at nodes $x$ and $y$ respectively, the graphs $A$ and $B, B$ and $A$ and $B$ and $B$ respectively. Then we have the following theorem. THEOREM 9. The graphs $\mathrm{H}_{1}, \mathrm{H}_{2}, \mathrm{H}_{3}$ and $\mathrm{H}_{4}$ all have the same matching polynomial $1 . e$. , $\left(\mathrm{H}_{1}, \mathrm{H}_{2}, \mathrm{H}_{3}, \mathrm{H}_{4}\right)$ is a comatching quadruple.

PROOF. The result can be established by applying the reduction process to the graphs until the attached subgraphs $A$ and $B$ become disconnected. This is accomplished by deleting the edges of $G$ which are incident to the nodes of attachment. The reduced graphs will either be $A$ or $B$. The incorporated graphs will be A - a, B - b and $\mathrm{G}-\{\mathrm{x}, \mathrm{y}\}$. The result will then follow from Lemma 3 .

6. DISCUSSION.

We have given several classes of comatching graphs. Also, we have given some general results which can be used to construct larger and larger classes from any given class. We have found other kinds of comatching graphs, but have not mentioned them here, since they do not appear to be very interesting.

\section{REFERENCES}

1. FARRELL, E. J. An Introduction to Matching Polynomials, J. Comb. Theory B, 27 (1979) 75-86. 


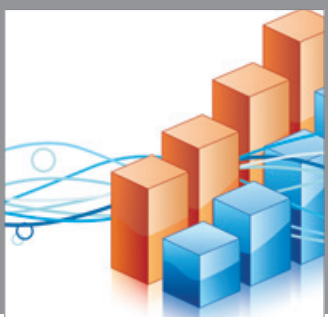

Advances in

Operations Research

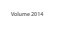

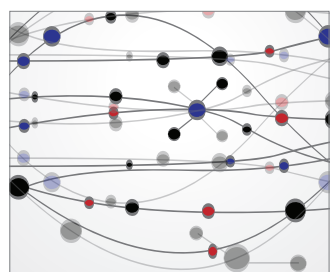

\section{The Scientific} World Journal
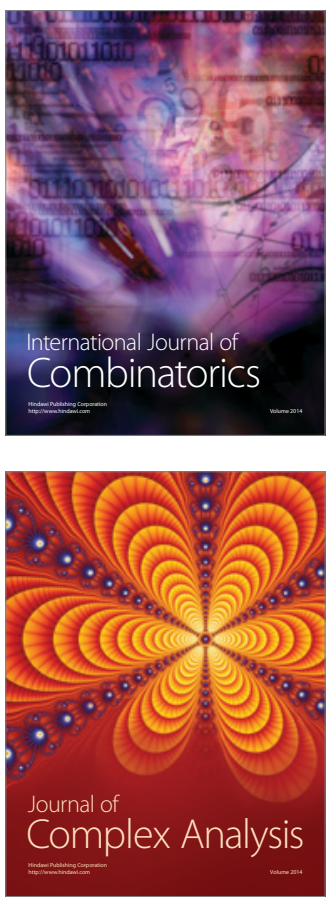

International Journal of

Mathematics and

Mathematical

Sciences
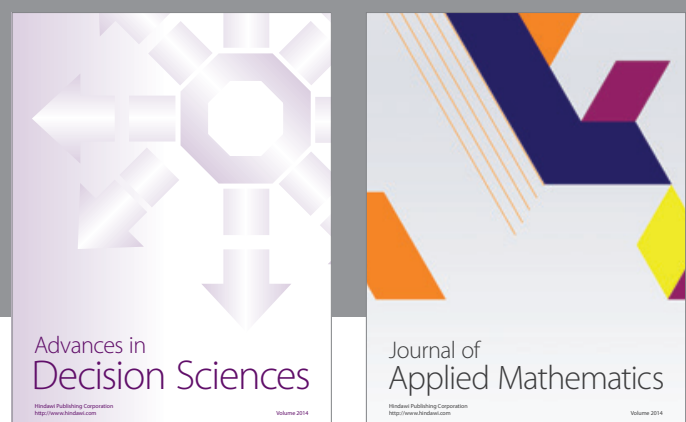

Journal of

Applied Mathematics
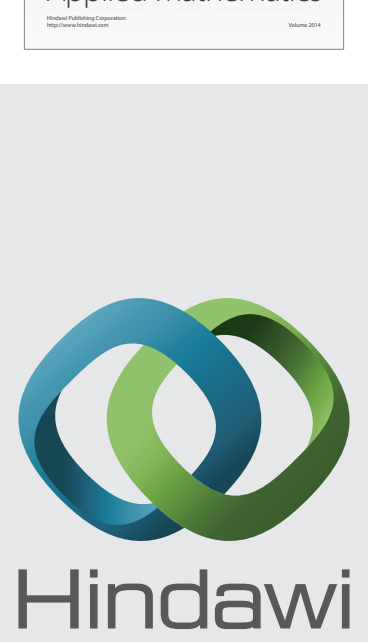

Submit your manuscripts at http://www.hindawi.com
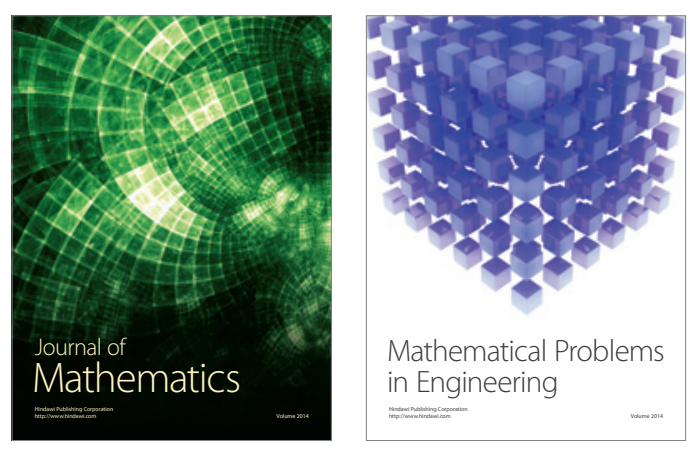

Mathematical Problems in Engineering
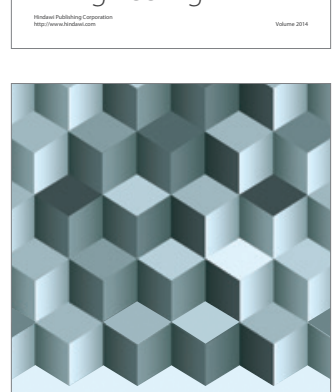

Journal of

Function Spaces
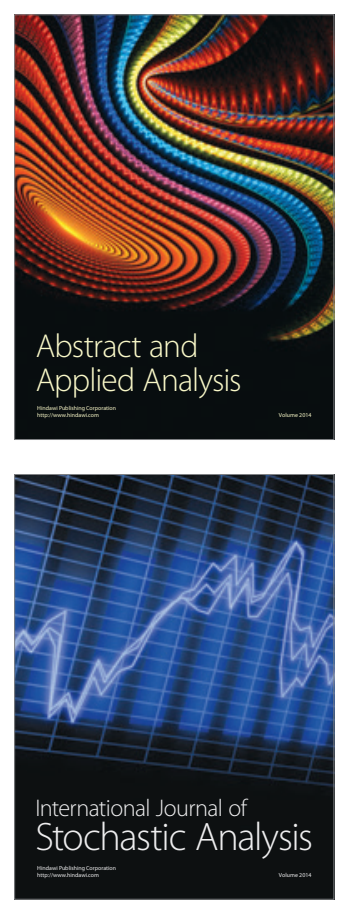

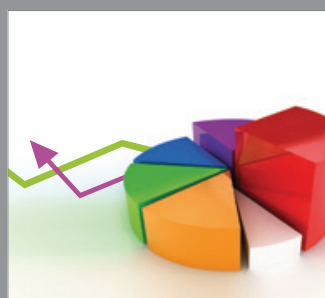

ournal of

Probability and Statistics

Promensencen
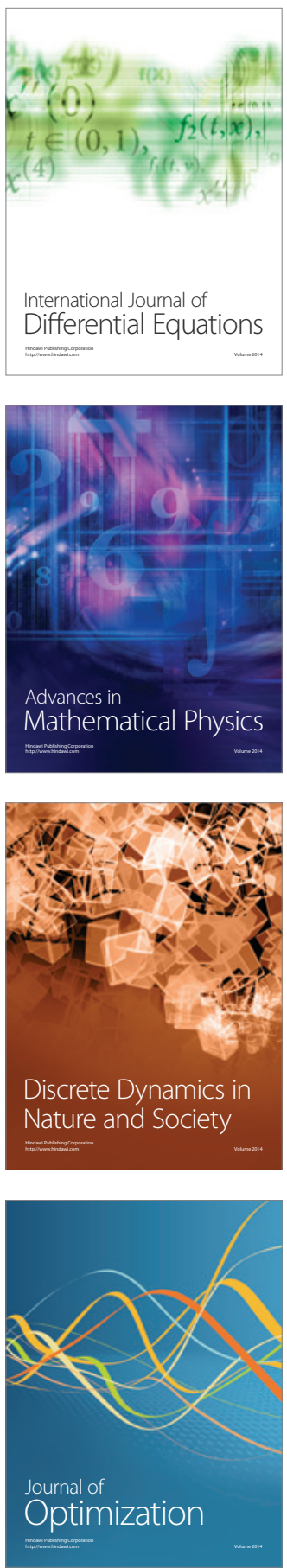